\title{
Move together, bond together: Visuomotor synchrony and social bonding in children and adults
}

4

Running title: Visuomotor synchrony and social bonding

\section{Corresponding Author:}

Ellen M. Howard

Ellen.howard@nottingham.ac.uk

School of Psychology,

The University of Nottingham,

Nottingham,

UK 
Interpersonal synchrony is a fundamental part of human social interaction, with known effects on facilitating social bonding. Moving in time with another person facilitates prosocial behaviour, however, it is unknown if the degree of synchronisation predicts the degree of social bonding. Similarly, while people readily fall in synchrony even without being instructed to do so, we do not know whether such spontaneous synchronisation elicits similar prosocial effects 33 as instructed synchronisation.

34 Across two studies, we investigated how context (social vs non-social stimulus) and instruction (instructed vs uninstructed) influenced synchronisation accuracy and bonding with the interaction partner in adults (Study 1; 34 women, 6 men, $M_{a g e}=20.38$, predominantly White undergraduates) and children (Study 2; 27 girls, 26 boys $M_{\text {age }}=8.80$, from predominantly

38 White middle-class families). The results revealed improved visuomotor synchrony within a 39 social, compared to non-social, context in adults and children. Children, but not adults, 40 synchronised more accurately when instructed to synchronise than when uninstructed. For both children and adults, synchronisation in a social context elicited stronger social bonding towards an interaction partner as compared to synchronisation in a non-social context. Finally, children's, but not adults', degree of synchrony with the partner was significantly associated with their feelings of social closeness. These findings illuminate the interaction of sensorimotor coupling and joint action in social contexts and how these mechanisms facilitate synchronisation ability and social bonding.

Keywords: interpersonal synchrony, social bonding, entrainment, visuomotor synchrony, mimicry 


\section{Move together, bond together: Visuomotor synchrony and social bonding} in children and adults

Interpersonal synchrony, whereby two or more people move in temporal and spatial coordination with each other, is observed frequently and cross-culturally in group dance, marching bands, and children's clapping games (Ehrenreich, 2006; McNeill, 1995). Research investigating embodied cognition has shown that synchronous interpersonal movement plays a crucial role in social bonding starting from early in infancy (Trainor \& Cirelli, 2015 Tunçgenç, et al., 2015; Tunçgenç \& Cohen, 2016). Although the ability to synchronise movements with external stimuli develops in early childhood years (Kurganksy \& Shupikova, 2011), relatively little is known about how a social versus non-social context impacts synchronisation accuracy and its subsequent social bonding outcomes in children and adults. Building upon bottom-up sensory and top-down joint action accounts of interpersonal synchrony, we examined the conditions that facilitate synchronisation and its social bonding outcomes in adults (Study 1) and in children (Study 2).

An individual can synchronise their movement with a non-social object (i.e., a metronome) or with another person (interpersonal synchrony). Research investigating interpersonal synchrony has found that individuals feel more bonded to others after performing a task with them that involves synchronous, as compared to asynchronous interpersonal movement. For instance, adults report increased feelings of cohesion, trust, empathy and prosocial behaviours following interpersonal synchrony (Anshel \& Kippler, 1988; Hove \& Risen, 2009; Launay et al., 2014; Valdesolo et al., 2010; Wiltermuth \& Heath, 2009). Similarly, in children, interpersonal synchrony has been shown to facilitate pro-social sharing (Rabinowitch \& Knafo-Noam, 2015; Rabinowitch \& Meltzoff, 2017) and helping behaviour (Tunçgenç \& Cohen, 2016). Therefore, moving in coordination with other people seems to incur social benefits. What, then, are the mechanisms that enable the positive social effects of movement synchrony? Two prominent accounts have been put forth in the literature to explain the synchrony - social bonding link: a bottom-up sensorimotor coupling account and a top-down joint action account.

Sensorimotor coupling defines the process whereby an external rhythm is identified (e.g., through visual observation) and integrated into one's own movements (Phillips-Silver et al., 2010). Previous research has shown that interpersonal synchrony is improved when 
visual and auditory (Gipson et al., 2016). Further, some stimuli may be easier to couple with than others. For instance, viewing a biologically similar stimulus (i.e., hand), compared to a non-social, mechanical stimulus, can facilitate interpersonal synchronisation (Calvo-Merino et al., 2005; Iacoboni et al., 1999, Kirschner \& Tomasello, 2009), arguably due to the ease of creating motor representations of the other person's actions (Brass \& Heyes, 2005; Rizzolatti, 2005; Casile et al., 2011). In turn, perceptual representation of synchronised movement may result in a less effortful and more rewarding experience through minimising neural processing costs (Ramamoorthy \& Konvalinka, 2017), thereby creating a cycle encouraging interpersonal synchrony. Indeed, an fMRI study has shown the brain's reward system to be activated following synchronous, as compared to asynchronous, interpersonal movement (Kokal et al., 2011). Thus, sensorimotor coupling plays a key role in interpersonal synchrony. Given that adults and children show greater social bonding to a partner following interpersonal synchrony, (e.g. Hove \& Risen, 2009; Tunçgenç \& Cohen, 2016) a social context that encourages sensorimotor coupling may facilitate synchronisation accuracy and social bonding.

In addition to perceptual and motor processes, top-down joint action processes are also thought to facilitate interpersonal synchrony. Joint action can be defined as the coordination or complementarity of two or more individuals" actions "to bring about a change in the environment" within a social interaction (Sebanz et al., 2006). According to this framework, the shared goal and mutual knowledge of the fact that this goal is shared unites the interaction partners' intentions, attention and representation of the task in hand (Tomasello et al., 2005). These shared representations enable easier prediction of the other's behaviour and movement coordination (Sebanz et al., 2006; Ramenozi et al., 2015). Prior research has indeed found that joint action contexts facilitate synchronisation, for instance when individuals shared the goal to synchronise with each other (van Ulzen et al., 2008). Even when individuals share a goal or intention that is not related to synchronisation, enhanced interpersonal synchrony can be observed. For example, individuals who shared a mutual goal to empty a box of 100 plastic balls coordinated movements more than those without a shared goal (Allsop et al., 2016). These works indicate that when individuals share a common goal, they are more likely to coordinate their movements with each other and thus joint action contexts can facilitate interpersonal synchrony. Developmentally, it was found that children as young as 2.5 years old synchronised better when performing a drumming activity with another person as opposed to with a robot (Kirschner \& Tomasello, 2009). Notably, the richness of visual information was comparable in the person and robot drummer conditions. Thus, the synchronisation boost observed in the 
116 person condition can be attributed to the ease of sensorimotor coupling with a biological 117 stimulus and/or sharing a representation of the "jointness" of the action. Although this study 118 shows that synchronisation improves in a condition in which both sensorimotor coupling and 119 joint action are facilitated simultaneously, it does not show whether social bonding outcomes 120 would follow. These highlighted works demonstrate how top-down processes, such as social 121 engagement in joint action contexts, can improve interpersonal synchrony. Thus, joint action context may also serve to boost synchronisation accuracy and social bonding.

In laboratory settings, interpersonal synchrony has often been studied in two conditions: by explicitly instructing the participants to move in synchrony with the target stimuli ("instructed synchrony") or by leaving the participants to move as they wish ("uninstructed synchrony"). Research has found that adults are better at walking in time to a non-social stimulus, such as an auditory beat, when they are explicitly instructed to do so (Leow et al., 2018). Furthermore, adults are more likely to tap in time to an auditory beat when they are instructed to do so (Repp \& Keller, 2004). These works suggest that intention to synchronise is highly influential for synchronisation with non-social objects. Considering interpersonal synchrony, studies have repeatedly shown that people tend to spontaneously entrain to others' movements when walking (Nessler et al., 2011), swinging their legs (Schmidt et al., 1990), clapping (Nèda et al., 2000) and rocking in chairs (Richardson et al., 2007). These findings suggest humans have a natural propensity to synchronise with others however, interpersonal synchrony can also be enhanced when an explicit instruction to synchronise is given (van Ulzen et al., 2008). Based on this research, we might expect that instructing participants to synchronise in either a social or non-social context would facilitate synchrony. Yet, no study has directly compared the effects of instruction within social and non-social contexts for synchronisation and its social bonding outcomes.

Given that our knowledge of social bonding effects is largely, if not entirely, based on research using instructed interpersonal synchrony (Anshel \& Kippler, 1988; Hove \& Risen, 2009; Launay et al., 2014; Valdesolo et al., 2010; Wiltermuth \& Heath, 2009), it is unclear whether uninstructed synchrony can elicit similar feelings of social bonding. Empirical

144 findings show that people have a tendency to fall into interpersonal synchrony with each other 145 (Nèda et al., 2000; Nessler et al., 2011; Richardson et al., 2007; Schmidt et al., 1990) yet we 146 do not know whether spontaneous interpersonal synchrony serves a social bonding function. It 147 is worth noting that when investigating interpersonal synchrony, the instructed and 
uninstructed conditions do not necessarily differ in terms of the degree of sensorimotor coupling or joint action context. When an individual is instructed to move in synchrony with another person, this goal is not necessarily shared between the two partners, and thus, a joint action context is not necessarily created. Whilst there is evidence that humans do have a natural propensity to synchronise with others, it is unknown whether similar social bonding effects are seen following uninstructed versus instructed synchrony.

Across two studies, we investigated how adults (Study 1) and children (Study 2) synchronise their movements and subsequently bond with their interaction partner.

156 Synchronisation was assessed with a finger-tapping task, with participants being randomly 157 assigned to either the instructed or uninstructed condition. Combining the bottom-up and top158 down accounts of interpersonal synchrony, we created a social context that included joint 159 action (i.e., participant tapping together with the partner) and better opportunities for 160 sensorimotor coupling (i.e., participant viewing the partner's hand). This social context was 161 contrasted to a non-social context that included neither joint action (i.e., participant tapping on their own while the partner is doing another task) nor enhanced sensorimotor coupling (i.e., participant viewing a moving ball).

164 We made four predictions: (1) participants will synchronise with the stimulus better in 165 the social than in the non-social context, (2) participants will feel more bonded to their partner 166 after completing the finger tapping task in a social as compared to a non-social context, (3) 167 synchrony and social bonding will be greater in the instructed compared to uninstructed condition, and (4) the degree of interpersonal synchrony in the social context will positively predict social bonding. The latter hypothesis would reveal the relative contributions of the topdown versus bottom-up processes - beyond performing a task in a joint action context, the precise degree of sensorimotor coupling would be associated with the social effects observed after interpersonal synchrony. 


\section{Methods}

176 Participants

177 Forty-three adults (37 women, 6 men, $\mathrm{M}_{\mathrm{age}}=20.44$ ), predominantly White, undergraduate 178 students, participated in the current study. Students volunteered to participate in return for 179 course credit. Data from three participants were removed due to experimenter error. Therefore, 180 the final sample consisted of forty adults (34 women, 6 men, $\mathrm{M}_{\mathrm{age}}=20.38$ ) evenly split into 181 the instructed and uninstructed conditions. Post-hoc power analysis and the reported effect sizes (see Results section below) show that despite some variability, our sample size allowed for sufficient power to detect the main effects found in both studies, when the type I error rate set was to $.05(1-\beta \geq .9)$. Ethical approval for both Study 1 and Study 2 were granted by the [masked for blind peer-review]. The experiment was conducted in accordance with the ethical standards of the Declaration of Helsinki and GDPR.

Design

A 2 (context: social vs non-social) x 2 (instruction type: instructed vs uninstructed) x 2 (stimulus tempo: $750 \mathrm{~ms}$ vs $1000 \mathrm{~ms}$ tempo) mixed design was used. All participants were presented with two social and two non-social trials, with the visual stimulus being presented at $750 \mathrm{~ms}$ and $1000 \mathrm{~ms}$ tempo within each condition. The two speeds (750ms and 1000ms) were used in order to maintain engagement with this repetitive task; no specific hypotheses were made about the effects of the speed conditions. The between-group factor was type of instruction, in which half of the participants were explicitly instructed to synchronise with the visual stimulus whilst the remaining half were told to tap as they wish, without any emphasis on synchronising with the stimulus. Participants were randomly assigned to the instructed or the uninstructed condition. Order of the social and non-social trials as well as the stimulus tempi was fully counterbalanced. The dependent variables were synchronisation accuracy (i.e., difference from the stimulus in ms) and social bonding as measured by subjective assessment of proximity with the partner.

\section{Materials and Measures}

Synchronisation accuracy. The tapping tasks were conducted using a mediated reality device called MIRAGE (see Figure 1A; Newport et al., 2010), which presents live video images of the participant's hand in real time. When participants put their hand into the MIRAGE device, they can no longer see their actual hand; instead, they see a live footage of their hand 
in the same spatial location, depth plane and from the same visual perspective as their 'real' hand. Each participant had their arm covered with a black curtain to prevent them from viewing their 'real' hand within the MIRAGE device. This device has been successfully used with adults and children in previous research (e.g. Greenfield et al., 2017; Newport \& Gilpin, 2011; Newport et al., 2015; Ropar et al., 2018).

In our study, the social (hand) and non-social (moving circle) stimuli were presented in the MIRAGE window opposite the live image of the participant's hand, as can be seen in Figure 1A. Finger taps were registered using a button placed under the participant's index finger. The MIRAGE device was connected to a computer that controlled the tempo of the visual stimulus and recorded the timestamps of the participants' taps at millisecond precision. Each participant completed four trials in total: two trials in the social context (at $750 \mathrm{~ms}$ and $1000 \mathrm{~ms}$ tempo) and two trials in the non-social context (at 750ms and 1000ms tempo). Each trial consisted of 70 cycles of visual stimulus. Therefore, for each participant, approximately 140 responses were recorded for the social context and 140 for the non-social context.

Synchronisation error was calculated as the Root Mean Square (RMS) of the difference between the participant's and the stimulus' inter-tap intervals. The stimulus inter-tap intervals were constant over time within a trial (i.e., either 750 or 1000 , depending on the stimulus tempo). To calculate the participants' inter-tap intervals, we subtracted the timestamp of one tap from the timestamp of the subsequent tap in a sliding window fashion (window size $=2$ ). Next, the difference between the participants' inter-tap intervals and that of the stimulus was taken. Finally, the RMS of this difference was calculated, yielding one synchronisation error score per participant per trial. To obtain single synchronisation accuracy scores for the social and non-social conditions each, we took the mean of the two trials comprising these conditions, respectively. Synchronisation error scores closer to zero indicate less difference between the participant and the stimulus, and thus better synchronisation accuracy.

Social closeness. Social closeness was measured using the 7-point Inclusion of Other in Self scale (Aron et al., 1992). In this scale, two circles labelled "you" and "[partner's name]" were shown in differing proximities to each other across the 7 points, starting with very distant at 1 and gradually getting closer, with a near-merging image at 7 . To calculate the change in feelings of closeness, a baseline measure was taken once at the start of the study and four more times at the end of each tapping trial. Change in social closeness was calculated separately for each experimental trial by deducting the participants' baseline score from their score following 
238 that trial. Then, the mean of the two difference scores obtained from the social and the non239 social trials were taken to construct one social and one non-social score per participant, 240 respectively. The more positive the social bonding difference values are, the closer the

241 participant felt towards the experimenter after the tapping task as compared to baseline.

242 Procedure

243 All participants were tested in a quiet room at [masked for blind peer-review], where 244 they were sat across from the experimenter and the partner in both social and non-social context 245 conditions (see Figure 1B for a schematic of the set-up). The experimental procedure took a 246 total of 30 minutes.

Firstly, participants completed a baseline measure of the Inclusion of Other in Self 248 scale. Then, participants placed their dominant hand into the MIRAGE to start the finger 249 tapping task. The participants were instructed to place the tip of their index finger on a button 250 that was used to register taps. The button made a dull click when tapped. The participants were 251 instructed to keep their hand in this location throughout all trials. All participants completed a 252 practice trial to ensure they were comfortable with MIRAGE and that they understood the task. 253 Once five consecutive taps had been successfully registered, the practice trial was terminated, 254 and the participant proceeded to the experimental trials. 
(A)

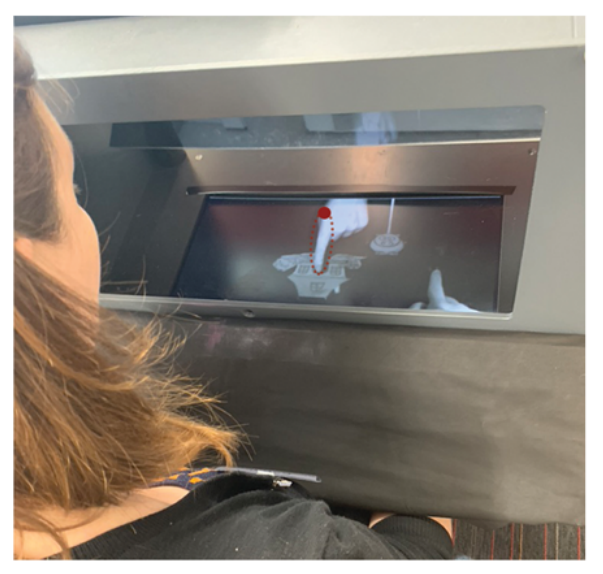

(B)

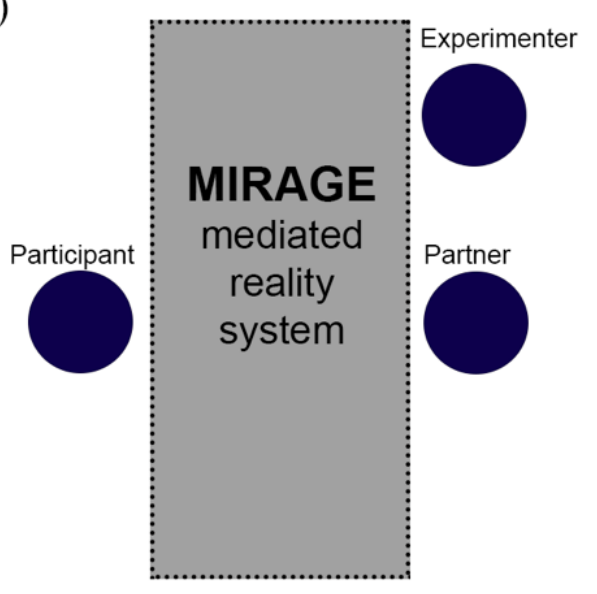

(C)

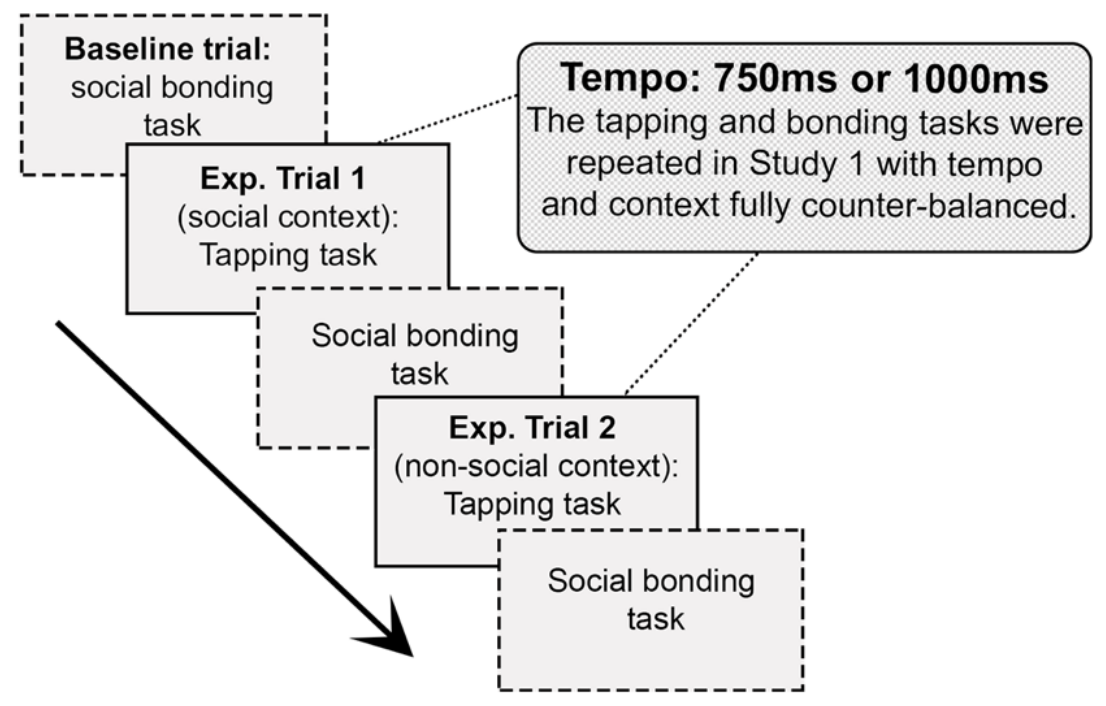

Figure 1. Schematic of the study procedure.

(A) Participant view of the MIRAGE window, with their hand on the bottom right of the window and the social stimulus (partner's hand) and non-social stimulus (red ball) opposite. The cartoon house and animal were used in the child-friendly dummy task.

(B) Study set-up. The partner sat in the same position in both the social and the non-social context conditions.

(C) Procedure of Study 1 and Study 2 (order of context and stimulus tempo counterbalanced across participants).

In the experimental trials, participants completed two social and two non-social context trials, each followed by the social closeness measure (see Figure 1C). Regardless of the

258 instruction condition, all participants were first given the following instructions: "If you look 259 through the MIRAGE window, you will see a ball (non-social context) moving elliptically up and down or [the partner's name]'s finger (social context) tapping. Please start tapping as soon as you see the ball/finger start and keep tapping until it stops". At this point, the instructions diverged depending on the instruction condition. While participants in the instructed condition were told "to try to tap in time with the ball/finger as best as you can", participants in the 
uninstructed condition were told "to keep looking at the ball/finger while tapping as slowly or as fast as you wish".

In the non-social context, a red ball moved up and down in an elliptical movement for the duration of the trial. In the social context, participants viewed a pre-recorded video of a

268 hand tapping although they were told that the partner was tapping in real time. The size of the

269 area in which the visual stimulus moved was kept constant across the social and non-social 270 stimuli to ensure that the amount of visual information was similar between conditions. In both 271 social and non-social context, the partner sat opposite the participant, without explicitly 272 interacting with the participant (e.g., by making eye contact or smiling). The only differences 273 between contexts were that in the social context: (a) the participants were told that the partner 274 was also finger-tapping, and (b) the participants viewed a hand, rather than a ball, as they 275 tapped.

To ensure that all participants attended to the stimuli similarly in all conditions, we also introduced a dummy task. A child friendly dummy task was used so it could also be introduced

278 in Study 2. Participants were told the visual stimulus was knocking on the door of the house 279 (see Figure 1A) and waking up the animals inside. Pictures of animals would then appear next 280 to the visual stimulus 2-3 times per trial. Since the animal pictures appeared next to the target 281 stimulus, participants were required to look towards the stimulus at all times and then tell the 282 names of the animals whose pictures popped up. 
Analysis

285

For both Study 1 and Study 2, the timestamp data was processed using a custom-written script on MATLAB R2018b and the statistical analyses (including the power analysis using package "pwr"; Champely et al., 2020) were conducted using R (R Core Team, 2014). To examine Hypotheses $1-3$, mixed 2 (context: social vs non-social - within variable) x 2 (instruction: instructed vs uninstructed - between groups variable) x 2 (stimulus tempo: $750 \mathrm{~ms}$ vs $1000 \mathrm{~ms}$ - within variable) separate ANOVA tests were conducted with synchronisation accuracy and social bonding measures as the dependent variables. Stimulus tempo was included in the analyses as an independent variable despite not being part of a specific hypothesis to control for its potential effects on our outcome measures. To examine Hypothesis 4, linear regression was conducted within the social context, with synchronisation accuracy as the predictor and social bonding measure(s) as the outcome variable(s).

\section{Synchronisation accuracy}

A mixed $2 \times 2 \times 2$ analysis of variance (ANOVA) was used to investigate the effects of context (social vs non-social), instruction (instructed vs uninstructed) and stimulus tempo (750ms vs $1000 \mathrm{~ms}$ ) on synchronisation accuracy (i.e., RMS of inter-tap intervals). The results revealed main effects of context $\left(F(1,37)=31.71, p<.0001\right.$, partial $\left.\eta^{2}=.46\right)$, and tempo $\left(F(1,37)=85.83, p<.0001\right.$, partial $\left.\eta^{2}=.70\right)$ on synchronisation accuracy. There was no significant main effect of instruction or any significant interactions (all $p \mathrm{~s}>.05$ ). Participants synchronised better (i.e., lower error) in the social context $(M=343.66, S D=103.04)$ than in the non-social context $(M=408.67, S D=97.38$; see Figure $2 \mathrm{~A})$ and better when the visual stimuli moved at $750 \mathrm{~ms}$ tempo $(M=319.81, S D=93.52)$ as compared to at $1000 \mathrm{~ms}$ tempo $(M$ $=431.70, S D=84.68)$. These findings show that irrespective of whether explicitly instructed to do so, participants were better able to synchronise with the partner's hand in the social context compared to the moving ball stimulus in the non-social context. To account for the possibility of more errors in cases when inter-tap intervals are larger (i.e., stimulus tempo at $1000 \mathrm{~ms}$ vs $750 \mathrm{~ms}$ ), we also calculated a normalised synchronisation accuracy metric by dividing the RMS inter-tap intervals by the stimulus tempo. Analyses using this normalised metric similarly showed significantly better synchrony performance in the social than in the non-social context (see Appendix A). 
(A)

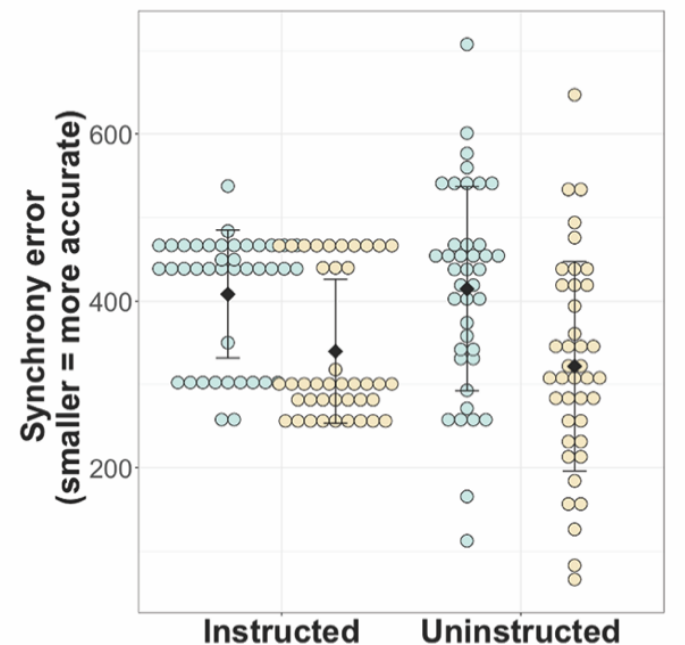

(B)

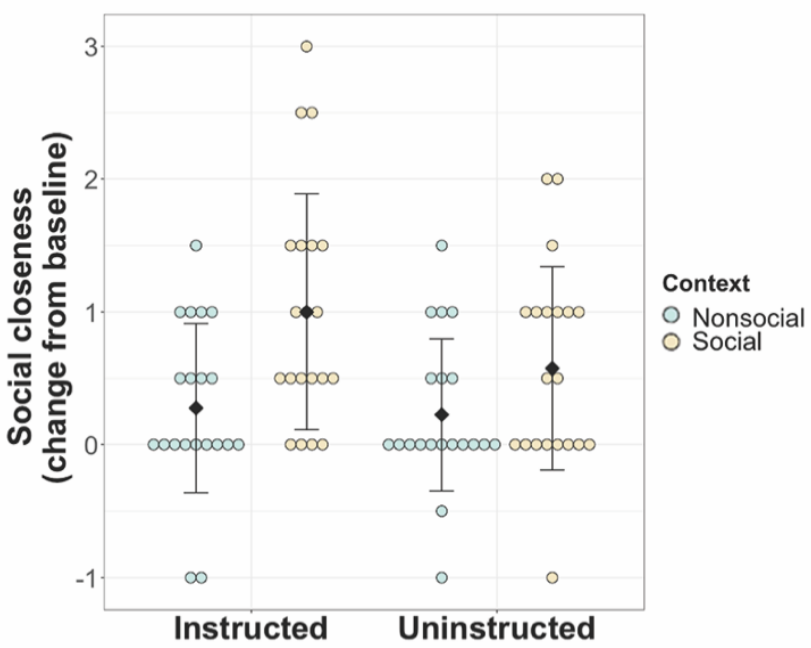

Figure 2. Synchronisation accuracy and social closeness by context and instruction conditions in adults. (A) Synchronisation accuracy per context and instruction condition. The y-axis shows mean RMS of the difference in inter-tap intervals between the participant and the stimulus, with higher scores indicating more errors and worse synchrony accuracy.

(B) Social closeness per context and instruction condition. Mean change in closeness score (test - baseline), with higher scores indicating an increase in closeness as compared to baseline.

Coloured dots indicate individual data points, black diamonds indicate group mean and whiskers indicate standard error of the mean.

\section{Social closeness}

To assess the impact of context (social vs non-social), stimulus tempo (750ms vs $1000 \mathrm{~ms}$ ), and instruction condition (instructed vs uninstructed) on change in social closeness

317 from baseline, a mixed $2 \times 2 \times 2$ ANOVA was employed. This analysis revealed a significant 318 main effect of context $\left(F(1,38)=32.49, p<.0001\right.$, partial $\left.\eta^{2}=.46\right)$, on social closeness. No 319 other main effects or interactions were statistically significant $(p>.05)$. While participants' social closeness towards the partner increased in the social context $(M=0.40, S D=1.09)$, a decrease was observed after doing the tapping activity in the non-social context $(M=-0.71, S D$

$322=0.9$ ), as can be seen in Figure 2B. This suggests that social context, but not explicit instructions to synchronise, affected the participants' feelings of social closeness.

Finally, using linear regression analysis, we explored whether synchrony accuracy is associated with change in social closeness within the social context condition. The results were not significant $(F(1,38)=0.92, p=.34, \beta=.003, S E=.003, p=.34)$, indicating that the degree of synchronisation with the partner was not related to the observed increase in feelings of social closeness. 


\section{Discussion}

As predicted, we found that a social context facilitated synchronisation accuracy and social bonding with an interaction partner in adults. This novel finding in adults corresponds to previous work that has demonstrated social facilitation of synchronisation accuracy in children (Kirschner \& Tomasello, 2009). Contrary to our predictions, synchronisation accuracy was not enhanced when the participants were explicitly instructed to tap in time with the stimulus, and the degree of interpersonal synchrony within the social context did not predict subsequent social bonding.

Despite the partner being present in both the social and non-social contexts, only the social context enhanced synchronisation accuracy and social bonding. This finding suggests that both synchronisation accuracy and social bonding can be facilitated (a) in a context in which the partner is perceived to be engaging in the task, and (b) when the participant views a biologically more similar stimulus that facilitates sensorimotor coupling. Given that our social context combined these two elements, we cannot identify specifically which aspect of the social context led to the observed effects. Nevertheless, these findings extend previous work that has found increased social bonding following instructed interpersonal synchrony (e.g., Hove \& Risen, 2009; Marsh et al., 2009; Launay et al., 2014) by showing that the same effects are observed following uninstructed synchrony as well.

The hypothesised effect of instruction on synchronisation accuracy was not observed; participants were similarly accurate at synchronising with the visual stimulus in the instructed and uninstructed conditions. Yet, visual inspection of the data shows that participant responses were less variable in the instructed condition than in the uninstructed condition, with the responses in the instructed condition displaying a bipolar distribution, suggesting a clustering of the errors based on the stimulus tempo. The absence of an effect of instruction seems to contradict previous work demonstrating that intentionality facilitates synchronisation (van Ulzen et al., 2008; Leow et al., 2018). However, in the current study, the intention was not explicitly shared between the participant and the partner, whilst in previous work the intention was shared between individuals (van Ulzen et al., 2008). This may suggest that shared intentionality between individuals, rather than individual intention, is key to facilitating coordination, rather than merely the instruction to synchronise.

Additionally, we found no relationship between the degree of synchronisation accuracy and the strength of feelings of social closeness. This may suggest that sensorimotor coupling of visual information did not drive the bonding effects; instead, joint action factors within the social context may have been sufficient to boost social closeness. 
363 Finally, despite not having any specific hypothesis based on stimulus tempo, we also

364 found that visuomotor synchronisation accuracy was higher with stimuli moving at a faster 365 tempo. This effect is most likely attributable to the Spontaneous Motor Tempo of individuals 366 being faster than the tempo at which the stimuli was presented. Previously work has found that 367 adults aged 18-38 years-old have a mean spontaneous motor tempo of 630ms (McAuley et al., 368 2006). It is therefore likely that participants were more comfortable tapping at the faster rate of $369750 \mathrm{~ms}$ than they were at $1000 \mathrm{~ms}$, therefore synchronisation accuracy was greater when the 370 tempo of the stimuli was faster. 
Participants

\section{Methods}

Sixty-one children (30 girls, 31 boys) aged 4.53 to 14.32 years old $\left(M_{\text {age }}=8.79, S D=\right.$ 2.18) from predominantly White, middle class families took part in the present study. The participants were recruited through a public event held at [masked for blind peer-review] in July 2019, in which children participate in short research studies over the course of a week. Three participants with a confirmed clinical diagnosis of Autism Spectrum Disorder were removed from analysis as previous work has found this population to exhibit atypical synchrony behaviour (Marsh et al., 2013; Fitzpatrick et al., 2016; Kaur et al., 2018). Four participants were removed from analysis for being extreme outliers ( \pm 2 SDs from the mean). Therefore, the final sample consisted of fifty-four neurotypical children (27 girls, 26 boys) aged 4.53 to 14.32 years old $\left(M_{\text {age }}=8.80, S D=2.16\right)$. There were 26 participants in the instructed condition and 28 participants in the uninstructed condition. All children were screened for developmental difficulties via a parental background questionnaire. Verbal mental age was assessed using the British Picture Vocabulary Scale III (BPVS III; Dunn \& Dunn, 2009) to ensure participants did not have a delay in their cognitive development. The parents of all children gave written consent for their child to participate and the children provided verbal assent at time of the study.

Design

The design of Study 2 differed slightly from Study 1 due to adjustments made for a child sample. Participants completed two, rather than four, tapping trials in total: one in the social context and one in the non-social context. This meant that stimulus tempo and the order of context conditions were counterbalanced across, not within, participants, such that half of the participants were presented with the social stimulus at $750 \mathrm{~ms}$ and the non-social at $1000 \mathrm{~ms}$, while the remaining half were presented with the social stimulus at $1000 \mathrm{~ms}$ and the non-social at $750 \mathrm{~ms}$. The independent variables of this study were identical to Study 2: context (social vs non-social), instruction type (instructed vs uninstructed) and stimulus tempo (750ms vs $1000 \mathrm{~ms}$ ). The dependent variables were synchronisation accuracy (i.e., difference from the stimulus in ms) and social bonding. Social bonding was measured in two ways: change in social closeness, measured by subjective assessment of proximity with the partner and change in 
mimicry task was added as a child-friendly way of assessing social bonding in addition to the proximity measure that mimicked the scale measure used in the adult study. The nature of the testing event in which Study 2 was conducted allowed for the addition of the spontaneous behavioural mimicry.

\section{Materials and Measures}

Synchronisation accuracy. To measure synchronisation accuracy, the same MIRAGE virtual reality device and the same measure of RMS of inter-tap intervals was used as in Study 1 .

Social closeness. A child-friendly version of the social closeness task was used here as compared to Study 1 to ensure participants could understand and relate to the task. To assess social closeness, the participants were asked to imagine a hypothetical scenario (e.g., waiting for a train at the station) and indicate first where they would like to sit, and then where they would like the partner to sit in this scenario by placing stickers that represented the child and the partner. The scenarios were accompanied by matching pictures that had 7 seats on them. Building upon similar measures that used physical closeness as a proxy for social closeness (Ijzerman \& Semin, 2010; Over \& Carpenter, 2015; Tuncgenc \& Cohen, 2016b), participants placing themselves closer to the partner was considered indicative of higher social closeness. Since the children were first asked to place themselves and then the partner, the distance between the two people was calculated as a proportion of the number of available seats once the children had placed themselves. To examine change in social closeness, the baseline distance score was subtracted from the distance scores following the tapping trials. Therefore, positive values indicated increased closeness from baseline, while negative values indicated decreased closeness.

Mimicry. Based on research showing positive affiliative effects of mimicking and being mimicked (for reviews, see: Lakin et al., 2003; Stel \& Vonk, 2010), spontaneous behavioural mimicry was assessed as a proxy for social bonding. Mimicry was assessed three times with a two-minute-long, semi-structured picture-guessing game played between the participant and the partner: first at baseline, and twice more thereafter each tapping trial. In the game, the partner viewed three pictures in sequence and described each to the participant. The participant, who had not seen the pictures, then guessed which picture (out of three options per picture) they thought the partner had been describing. Whilst describing the pictures, the 
435

436

437

438

439

440

441

442

443

444

445

446

447

448

449

450

451

452

453

454

455

456

457

458

459

460

461

462

463

464

465

partner made upper body touches on three areas (i.e., rubbing or scratching her head, shoulder or upper arm), amounting to a total of nine touches per block. The same picture descriptions were used across participants with the touches occurring at the same timepoints.

The frequency of spontaneous behavioural mimicry was post-hoc coded from the video recordings of the mimicry sessions using the open-source E-LAN software (version 5.8). An action was considered an instance of mimicry if the children touched, rubbed or scratched their head, shoulders or upper arms following the partner performing one of these actions within the same block. Two raters, one blind to the hypotheses and both blind to the actions of the partner, coded the videos. Inter-rater reliability on $40 \%$ of the videos was excellent $(r(25)=.89, p<$ $.0001)$. To examine change in mimicry, difference scores were obtained by deducting the frequency of mimicry at baseline from the frequency of mimicry following each tapping trial.

\section{Procedure}

The procedure was identical to Study 1 with the addition of the spontaneous behavioural mimicry measure completed at baseline and following each tapping trial.

\section{Synchronisation accuracy}

\section{Results}

A mixed 2x2x2 ANOVA was used to investigate the effects of context (social vs non-social within variable), instruction (instructed vs uninstructed - between groups variable) and stimulus tempo (750ms vs $1000 \mathrm{~ms}$ - within variable) on synchronisation accuracy (i.e., RMS of inter-tap intervals). The results revealed significant main effects of context $(F(1,48)=9.56$, $p=.003$, partial $\left.\eta^{2}=.17\right)$, instruction $\left(F(1,49)=84.11, p<.000\right.$, partial $\left.\eta^{2}=.63\right)$ and tempo $\left(F(1,48)=9.89, p=.003\right.$, partial $\left.\eta^{2}=.17\right)$. As can be seen in Figure 3A, participants' synchronisation accuracy was higher (i.e., lower error) in the social context $(M=295.16, S D$ $=205.9)$ than in the non-social context $(M=343.53, S D=227.75)$. Participants also synchronised better in the instructed condition $(M=154.22, S D=87.27)$ than in the uninstructed condition $(M=452.06, S D=188.07)$ and when the stimulus moved at $750 \mathrm{~ms}(M$ $=290.16, S D=195.57)$ than at $1000 \mathrm{~ms}(M=352.92, S D=237.63)$. There was a significant interaction between context and tempo $F(1,48)=35.19, p<.0001$ (see Figure 3B), such that within the $1000 \mathrm{~ms}$ tempo, synchronisation accuracy was better in the social $(M=268.59, S D$ $=188.41)$ than in the non-social $(M=443.99, S D=254.76)$ context $(F(1,22)=34.07, p<$ $.0001)$, while no such difference was observed within the 750ms tempo $(p=.56)$. 
466 Similar to the approach followed in Study 1, we also assessed the normalised synchronisation 467 accuracy metric, which was calculated by dividing the RMS of inter-tap intervals by the 468 stimulus tempo for that trial. These results confirmed that even when accounting for the 469 possibility of increased errors in larger inter-tap intervals, the effect of context remained 470 significant such that children synchronised with the stimulus better in the social than in the 471 non-social context (see Appendix B).

(A)

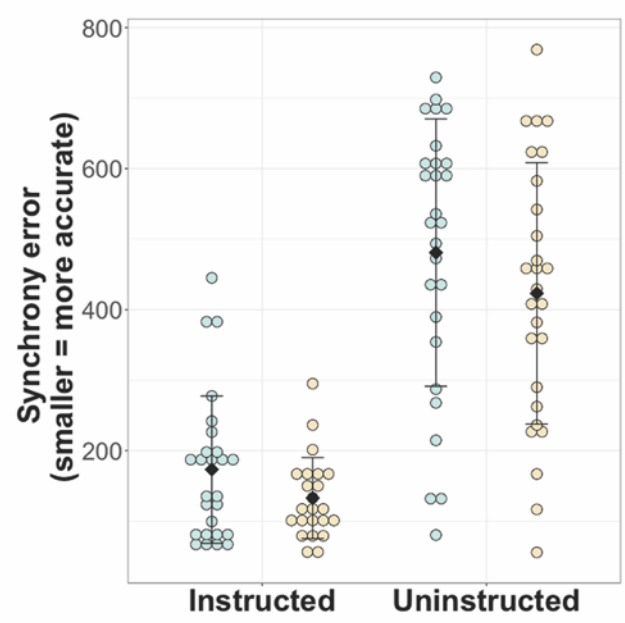

(B)

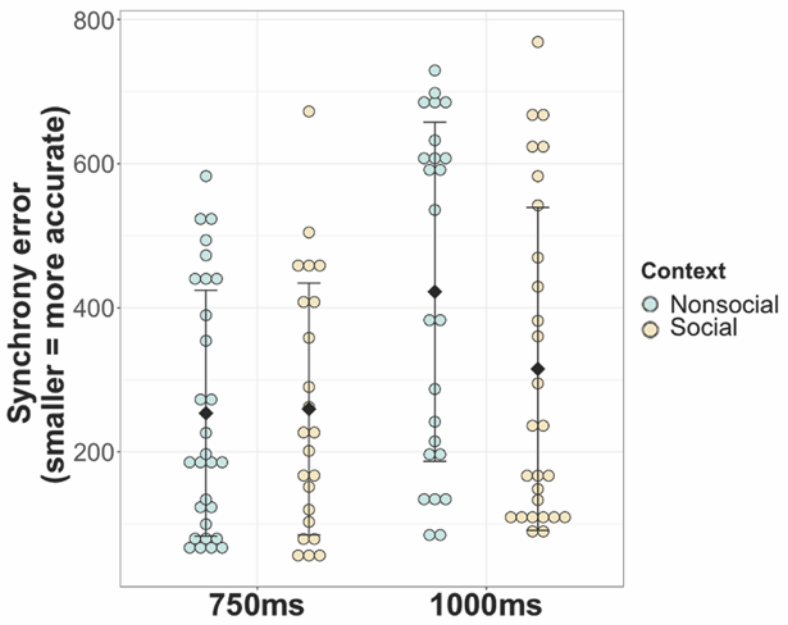

Figure 3. Synchrony accuracy by context, stimulus tempo and instruction conditions in children.

Lower scores on the y axis indicate less synchrony errors and better accuracy. Coloured dots indicate individual data points, black diamonds indicate group mean and whiskers indicate standard error of the mean.

(A) Synchronisation accuracy per context and instruction condition.

(B) Synchronisation accuracy per context and stimulus tempo conditions.

472

473

474

475

476

477

478

479

480

481

\section{Social closeness}

A mixed $2 \times 2 \times 2$ ANOVA was used to examine the effects of context (social vs nonsocial), instruction (instructed vs uninstructed) and stimulus tempo (750ms vs $1000 \mathrm{~ms}$ ) on change in social closeness (i.e., difference from baseline). This analysis revealed a significant main effect of context on social closeness $\left(F(1,44)=9.38, p=.004\right.$, partial $\left.\eta^{2}=.18\right)$. There was no significant main effect of instruction or stimulus tempo and no interaction effects (all $p$ 's $>$ .05). As can be seen in Figure 4A, while social closeness towards the partner increased following tapping in the social context $(M=2.13, S D=37.41)$, a decrease was observed in feelings of closeness following tapping in the non-social context $(M=-14.85, S D=37.69)$. 

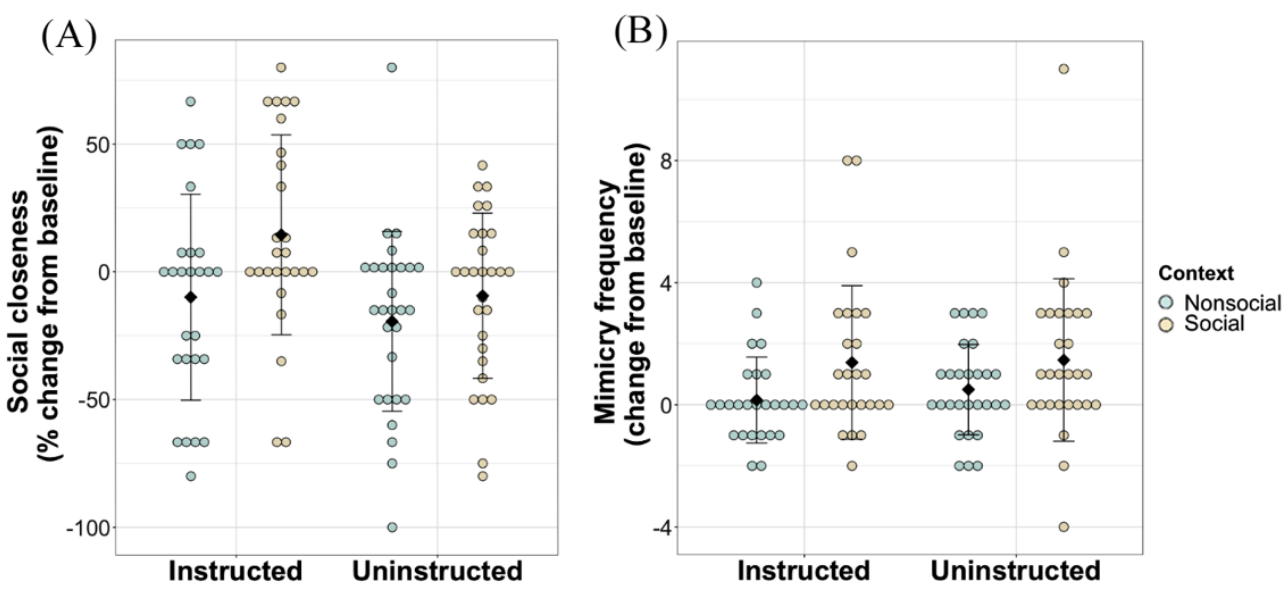

(C)

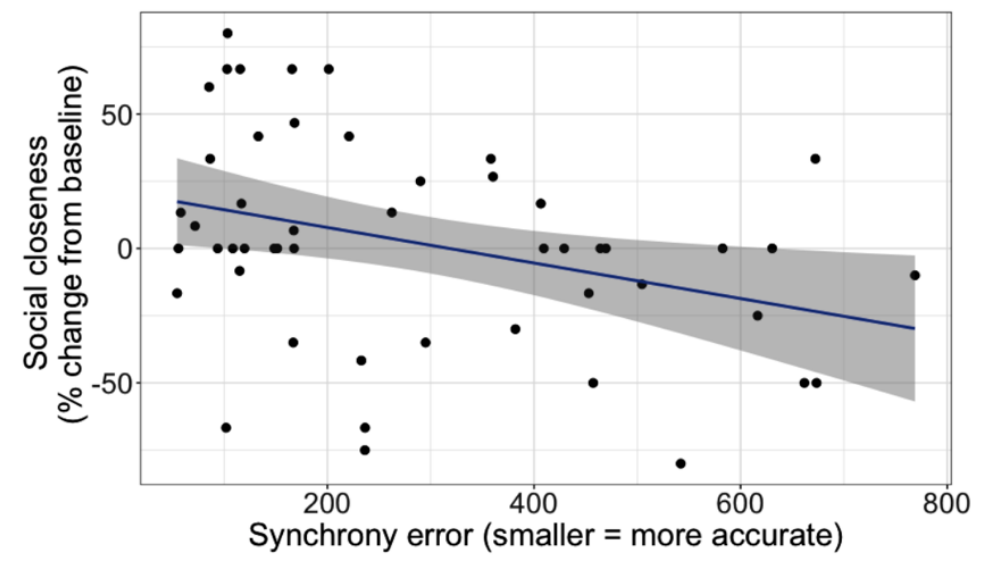

Figure 4. Social bonding by context, instruction and synchronisation accuracy in children.

In (A) and (B), coloured dots indicate individual data points, black diamonds indicate group mean and whiskers indicate standard error of the mean.

(A) Change in social closeness (test - baseline) per context and instruction condition, with positive scores indicating an increase and negative scores indicating a decrease in closeness as compared to baseline.

(B) Change in mimicry frequency (test - baseline) per social context and instruction condition, with positive scores indicating an increase and negative scores indicating a decrease in mimicry as compared to baseline.

(C) Relationship between synchrony error and change in social closeness within the social context.

Mimicry

A mixed $2 \times 2 \times 2$ ANOVA was used to investigate the effects of context (social vs nonsocial), instruction (instructed vs uninstructed) and stimulus tempo (750ms vs $1000 \mathrm{~ms})$ on

495 change in mimicry frequency (i.e., difference from baseline). The results revealed a main effect 496 of context on mimicry frequency $\left(F(1,44)=19.93, p<.0001\right.$, partial $\left.l \eta^{2}=.31\right)$. There was no 497 significant main effect of instruction, stimulus tempo or any interaction effects (all $p$ 's $>.05$ ). 498 As can be seen in Figure 4B, while mimicry frequency showed an increase from baseline in 499 both social and non-social context conditions, this increase was significantly greater in the 500 social context $(M=1.72, S D=2.69)$ as compared to the non-social context $(M=0.34, S D$ $501=1.47$ ). 

synchronisation accuracy within the social context condition predicted change in spontaneous behavioural mimicry and social closeness. While synchronisation accuracy did not predict change in mimicry frequency $(F(1,45)=0.29, p=.59, \beta=.001, S E=.002, p=.59)$, it significantly predicted change in social closeness $(F(1,48)=20.47, p<.0001, \beta=.08, S E=$ $.02, p<.0001)$. Pearson correlation test similarly showed a significant negative relationship between synchrony errors and social closeness $(r(48)=-.34, p=.01$; see Figure $4 \mathrm{C})$. Therefore, as synchrony between the children and the partner increased, so did the children's subsequent feelings of social closeness.

\section{Discussion}

512

As predicted, and in line with previous works (Kirschner \& Tomasello, 2009; Tunçgenç et al., 2015), we found that both visuomotor synchrony and social bonding were facilitated in a social as compared to a non-social context. Extending previously used measures of social bonding, we showed that children spontaneously mimicked their partner more following interpersonal synchrony compared to synchronisation in the non-social context.

In line with Hypothesis 3, children synchronised with significantly greater accuracy when explicitly instructed to synchronise with the stimulus compared to when they were left free to tap as they wished. Unlike the adults in Study 1, children may have had a heightened sense of commitment when instructed, and hence increased effort to synchronise (Michael \& Szekely, 2018). In addition, prior work has demonstrated children are less consistent in how they tap as compared to adults (Geuze \& Kalverboer, 1994; Greene \& Williams, 1993), so it is possible that an explicit instruction therefore increased effort and improved synchronisation accuracy in the instructed condition.

Additionally, despite lower synchronisation in the uninstructed condition, social bonding for both measures remained similar across uninstructed and instructed conditions. This may suggest that merely completing the task together with the partner allowed for social bonding to occur. Yet, in line with Hypothesis 4, the degree of interpersonal synchrony still mattered: the more synchronous the children were with the partner, the greater was their feelings of social closeness. In comparison to adults, this may suggest that children are more likely to base social bonding on unconscious precise sensorimotor coupling between themselves and a partner. 
Similar to the adult data, we found an unexpected effect of stimulus tempo on synchronisation accuracy. It is likely that a faster spontaneous motor tempo of children led to greater accuracy in the $750 \mathrm{~ms}$ tempo compared to the $1000 \mathrm{~ms}$ tempo (McAuley et al., 2006). facilitated interpersonal synchrony and social bonding in both adults (Study 1) and children (Study 2). Uniquely in children, we found that magnitude of social closeness was related to the degree of synchronisation accuracy.

We found evidence of social context facilitating interpersonal synchrony in adults and children. Previous paradigms have been criticised for their use of social and non-social contexts as often the social condition holds more sensory information, therefore allowing for more accurate synchronisation (see Kirschner \& Tomasello, 2009). For instance, the available sensory information is much richer viewing the movement of a whole body as compared to a flashing dot or metronome. However, in the current study only the image of a hand was used as the social context, therefore the area covered by the moving visual stimuli was the same between conditions. Subsequently, this social facilitation effect is unlikely to be attributable to the amount of visual information. Instead, viewing a finger tapping may have enhanced neural representation of the external stimulus (i.e., hand movement), therefore increasing predictability and coordination with the stimulus, (Brass \& Heyes, 2005; Calvo-Merin et al., 2005; Iacoboni et al., 1999) making it easier to synchronise with. According to predictive coding principles, synchronisation with the social stimulus may have been less cognitively demanding and effortful, therefore producing fewer errors (Koban et al., 2019). Furthermore, this current finding corresponds to previous works that have suggested the presence of a social agent can induce perceptions of a joint action (Kirschner \& Tomasello, 2009; Tunçgenç et al., 2015). Such joint action formation is argued to allow for better anticipation of movement of the external stimulus (Sebanz et al., 2006) and therefore give rise to a coupling of self and other movement (Hove, 2008).

Our social context differed from the non-social context both in terms of providing better opportunities for participants to couple with the visual stimulus (i.e., with a hand as opposed to a ball), and in terms of the partner engaging in joint action with the participants. Both of these factors may facilitate social bonding through promoting participants' perception of the partner as moving like them and being like them (Meltzoff, 2007). We found that in adults, the degree of synchronisation accuracy did not predict subsequent feelings of social closeness, 
while in children it did. This may suggest that sensorimotor coupling was not fundamental for adults to feel social closeness; instead, the joint action context of the social condition may have been sufficient to enhance social bonding. Alternatively, as the accuracy of synchronisation was not consequential to task success, it may have reduced the extent that accuracy impacted upon feelings of social closeness. Future research can create experimental conditions that disentangle the relative roles of bottom-up and top-down factors in facilitating social bonding following synchrony.

In addition, children mimicked the partner more following interpersonal synchronisation. Whilst this corresponds to previous research that has shown mimicry can facilitate social bonding (Lakin et al., 2003; Stel \& Vonk, 2010), the present finding shows for the first time that synchronisation in a joint action context can elicit increased mimicry. This expands possible measures of social bonding to include mimicry. Although interpersonal synchrony enhanced both social closeness and mimicry, the two aspects are distinguishable as synchronisation accuracy did not predict a change in mimicry. One reason for this may be the relatively reduced variability in the mimicry frequencies compared to the social closeness scores, yielding a correlational approach less powerful. Still, the absence of a link between mimicry and interpersonal synchrony suggests that while social closeness may be more selectively boosted by interpersonal synchrony compared, merely completing the tapping task with the partner appears to be sufficient for increased mimicry to occur.

This study has emphasised the importance of interpersonal synchrony for social bonding, which opens up further questions as to whether an impaired ability to synchronise, especially in social contexts, may be related with social-communicative impairments. For instance Autism Spectrum Conditions are characterised by both socio-communication (Wing \& Gould, 1979) and sensorimotor differences (Greenfield et al., 2017; Ropar et al., 2018). Future work could therefore explore visuomotor synchrony in autistic children and adults.

Whilst the present study provides key insights into the roles of instruction, context, and tempo on visuomotor synchronisation and social bonding, certain limitations should be noted. Firstly, in Study 2 not all participants experienced both contexts at both tempi due to time constraints of the event at which the experiment was conducted. While it is unlikely that groups of children would be distinguished by their ability to synchronise better at social-fast vs socialslow stimuli, thereby affecting the results in a systematic way, future research can implement full counterbalancing to eliminate this possibility. Finally, the social vs non-social contrast in the current study was created by combining insights from the bottom-up sensorimotor accounts 
602 with the top-down joint action accounts. Future research can tease apart how either factor 603 affects synchrony accuracy and its subsequent bonding effects.

604 The current research adds to a growing body of literature by revealing the importance 605 of social context for interpersonal synchronisation and social bonding in children and adults. 606 For the first time, we show how social bonding can be elicited following spontaneous 607 (uninstructed) interpersonal synchronisation. These findings provide an evidence-base that 608 social contexts with increased opportunities for sensorimotor coupling and joint action improve 609 synchronisation and bonding between partners. 
611 Conceived and designed the experiment: [masked for blind peer-review]. Performed the

612 experiments: [masked for blind peer-review]. Analysed the data: [masked for blind peer-

613 review]. Wrote the paper: [masked for blind peer-review]. Edited the paper: [masked for

\section{Acknowledgements}

Ellen Howard was supported by an Economic and Social Research Council (ESRC) PhD Studentship (grant number ES/P000711/1). The funders had no role in the study design, data collection, analysis, decision to publish or preparation of the manuscript.

We would like to thank Lauren Fingret and Lucy Drage for their help in data collection for Study 1 .

624

625

626

627

628

629

630

631

632

633

634

635

636

637

638

639

640

641

642

643

644

645

646

647

648

\section{References}

Alderisio, F., Fiore, G., Salesse, R. N., Bardy, B. G., \& Bernardo, M. Di. (2017). Interaction patterns and individual dynamics shape the way we move in synchrony. Scientific Reports, 7(1). doi.org/10.1038/s41598-017-06559-4

Allsop, J. S., Vaitkus, T., Marie, D., \& Miles, L. K. (2016). Coordination and collective performance: Cooperative goals boost interpersonal synchrony and task outcomes. Frontiers in Psychology, 7(SEP), 1-11. doi.org/10.3389/fpsyg.2016.01462

Anshel, A., \& Kippler, D. (1988). The influence of group singing on trust and cooperation. Journal of Music Therapy, 25, 145-155. doi.org/10.1093/jmt/25.3.145

Aron, A., Aron E. N., \& Smollan, D. (1992). Inclusion of other in the self scale and the structure of interpersonal closeness. Journal of Personality and Social Psychology, 63, 596-612. doi.org/10.1037/0022-3514.63.4.596

Brass, M., \& Heyes, C. (2005). Imitation: Is cognitive neuroscience solving the correspondence problem? Trends in Cognitive Sciences, 9, 489-495. dx.doi.org/10.1016/j.tics.2005.08.007

Calvo-Merino, B., Glaser, D. E., Grèzes, J., Passingham, R. E., \& Haggard, P. (2005). Action observation and acquired motor skills: An FMRI study with expert dancers. Cerebral Cortex, 15, 1243-1249. dx.doi.org/ 10.1093/cercor/bhi007

Casile, A., Caggiano, V., \& Ferrari, P. F. (2011). The Mirror Neuron System: A Fresh View Antonino. Neuroscientist, 17(5), 524-538. doi.org/10.1038/jid.2014.371

Champely, S., Ekstrom, C., Dalgaard, P., Gill, J., Weibelzahl, S., Anandkumar, A., Ford, C., Volcic, R. \& De Rosario, H., (2020). pwr: Basic Functions for Power Analysis. $R$ package version 1.3-0. CRAN.R-project.org/package $=$ pwr

Cirelli, L. K., Einarson, K. M., \& Trainor, L. J. (2014). Interpersonal synchrony increases prosocial behavior in infants. Developmental Science, 17(6), 1003-1011. doi.org/10.1111/desc. 12193 
661

662

663

664

665

666

667

668

669

670

671

672

673

674

675

676

677

678

679

680

681

682

683

684

685

686

687

Dunn, L. M., \& Dunn, D. M. (2009). The British picture vocabulary scale. GL Assessment Limited.

Ehrenreich, B. (2006). Dancing in the streets. New York: Metropolitan. doi.org/10.2752/175183408x328370

ELAN (Version 5.8) [Computer software]. (2019). Nijmegen: Max Planck Institute for Psycholinguistics, The Language Archive. Retrieved from archive.mpi.nl/tla/elan

Fitzpatrick, P., Frazier, J. A., Cochran, D. M., Mitchell, T., Coleman, C., \& Schmidt, R. C. (2016). Impairments of social motor synchrony evident in autism spectrum disorder. Frontiers in Psychology, 7(AUG), 1-13. doi.org/10.3389/fpsyg.2016.01323

Geuze, R. H., \& Kalverboer, A. F. (1994). Tapping a rhythm: A problem of timing for children who are clumsy and dyslexic? Adapted Physical Activity Quarterly, 11, 203-213. doi.org/10.1123/apaq.11.2.203

Gipson, C. L., Gorman, J. C., \& Hessler, E. E. (2016). Top-down (prior knowledge) and bottom-up (perceptual modality) influences on spontaneous interpersonal synchronization. Nonlinear Dynamics, Psychology, and Life Sciences, 20(2), 193-222.

Greene, L. S., \& Williams, H. G. (1993). Age-related differences in timing control of repetitive movement: Application of the Wing-Kristofferson model. Research Quarterly for Exercise and Sport, 64, 32-38. doi.org/10.1080/02701367.1993.10608776

Greenfield, K., Ropar, D., Themelis, K., Ratcliffe, N., \& Newport, R. (2017). Developmental changes in sensitivity to spatial and temporal properties of sensory integration underlying body representation. Multisensory Research, 30(6), 467-484. doi.org/10.1163/22134808-00002591

Hove M. J. (2008). Shared circuits, shared time, and interpersonal synchrony. Behav. Brain Sci. 31, 29-30. doi:10.1017/S0140525X07003202

Hove, Michael, J., \& Risen, Jane, L. (2009). It's All in the Timing : Interpersonal Synchrony Increases Affiliation. Social Cognition, 27(6), 949-961. doi.org/10.1521/soco.2009.27.6.949

Iacoboni, M., Woods, R. P., Brass, M., Bekkering, H., Mazziotta, J. C., \& Rizzolatti, G. (1999). Cortical mechanisms of human imitation. Science, 286, 2526-2528. doi:10.1126/science.286.5449.2526

Ijzerman, H., and Semin, G. R., (2010). Temperature perceptions as a ground for social proximity. J. Exp. Soc. Psychol. 46, 867-873. doi: 10.1016/j.jesp.2010.07.015

Kaur, M., M. Srinivasan, S., \& N. Bhat, A. (2018). Comparing motor performance, praxis, coordination, and interpersonal synchrony between children with and without Autism Spectrum Disorder (ASD). Research in Developmental Disabilities, 72(February 2018), 79-95. doi.org/10.1016/j.ridd.2017.10.025

Kirschner, S., \& Tomasello, M. (2009). Joint drumming: Social context facilitates synchronization in preschool children. Journal of Experimental Child Psychology, 102(3), 299-314. doi.org/10.1016/j.jecp.2008.07.005 
Knoblich, G., \& Sebanz, N. (2008). Evolving intentions for social interaction: From entrainment to joint action. Philosophical Transactions of the Royal Society B: Biological Sciences, 363(1499), 1166-1177. doi.org/10.1098/rstb.2008.0006

Koban, L., Ramamoorthy, A., \& Konvalinka, I. (2019). Why do we fall into sync with others? Interpersonal synchronization and the brain's optimization principle. Social Neuroscience, 14(1), 1-9. doi.org/10.1080/17470919.2017.1400463

Kokal, I., A. Engel, S. Kirschner \& C. Keysers. 2011. Synchronized drumming enhances activity in the caudate and facilitates prosocial commitment - if the rhythm comes easily. PLoS One 6: e27272. doi: 10.1371/journal.pone.0027272.16.

Kurgansky, A. V., \& Shupikova, E. S. (2011). Visuomotor synchronization in adults and sevento eight-year-old children. Human Physiology, 37(5), 526-536. doi.org/10.1134/S0362119711050100

Lakin J.L., Jefferis V.E., Cheng C.M., \& Chartrand T.L. (2003) The chameleon effect as social glue: evidence for the evolutionary significance of nonconscious mimicry. J Nonverbal Behav 27(3):145-162. doi.org/10.1023/A:1025389814290

Launay, J., Dean, R. T., \& Bailes, F. (2014). Synchronising movements with the sounds of a virtual partner enhances partner likeability. Cognitive Processing, 15(4), 491-501. doi.org/10.1007/s10339-014-0618-0

Marsh, K. L., Isenhower, R. W., Richardson, M. J., Helt, M., Verbalis, A. D., Schmidt, R. C., $\&$ Fein, D. (2013). Autism and social disconnection in interpersonal rocking. Frontiers in Integrative Neuroscience, 7(JAN), 1-8. doi.org/10.3389/fnint.2013.00004

Marsh, K. L., Richardson, M. J., \& Schmidt, R. C. (2009). Social Connection Through Joint Action and Interpersonal Coordination. Topics in Cognitive Science, 1(2), 320-339. doi.org/10.1111/j.1756-8765.2009.01022.x

McAuley, J. D., Jones, M. R., Holub, S., Johnston, H. M., \& Miller, N. S. (2006). The time of our lives: Life span development of timing and event tracking. Journal of Experimental Psychology: General, 135(3), 348-367. doi.org/10.1037/0096-3445.135.3.348

McNeill, W. (1995). Keeping together in time: Dance and drill in human history. Cambridge, MA: Harvard University Press. doi.org/10.2307/j.ctvjf9wq6

Meltzoff A. N., (2007). 'Like me': a foundation for social cognition. Developmental science. 10: 126-134. doi: 10.1111/j.1467-7687.2007.00574.x

Michael, J., \& Székely, M. (2018). The Developmental Origins of Commitment. Journal of Social Philosophy, 49(1), 106-123. doi.org/10.1111/josp.12220

Néda, Z., Ravasz, E., Brechet, Y., Vicsek, T., \& Barabási, A. L. (2000). The sound of many hands clapping. Nature, 403 (6772), 849-850. doi:10.1038/35002660

Nessler, J. A., Gonzales, T., Rhoden, E., Steinbrick, M., \& De Leone, C. J. (2011). Stride interval dynamics are altered when two individuals walk side by side. Motor Control, 15(3), 390-404. doi.org/10.1123/mcj.15.3.390 
Newport, R., \& Gilpin, H. R. (2011). Multisensory disintegration and the disappearing hand trick. Current Biology, 21(19), R804-R805. doi.org/10.1016/j.cub.2011.08.044

Newport, R., Auty, K., Carey, M., Howard, E. M., Greenfield, K., Ratcliffe, N., Thair, H., \& Themelis, K. (2015). Give it a tug and feel it grow: Extending body perception through the universal nature of illusory finger stretching. I-Perception, 6(5), 1-4. doi.org/10.1177/2041669515599310

Noy, L., Dekel, E., \& Alon, U. (2011). The mirror game as a paradigm for studying the dynamics of two people improvising motion together. Proceedings of the National Academy of Sciences of the United States of America, 108, 20947-20952. doi:10.1073/pnas.1108155108

Oullier, O., de Guzman, G. C., Jantzen, K. J., Lagarde, J., \& Kelso, J. A. S. (2008). Social coordination dynamics: Measuring human bonding. Social Neuroscience, 3(2), 178192. doi:10.1080/17470910701563392

Over, H., \& Carpenter, M. (2015). Children infer affiliative and status relations from watching others imitate. Developmental Science, 18(6), 917-925. doi.org/10.1111/desc.12275

Phillips-Silver, J., Aktipis, C. A., \& Bryant, G. A. (2010). Entrainment, foundations of coordinated rhythm. Music Perception, 28(1), 3-14. doi.org/10.1525/mp.2010.28.1.3.

Rabinowitch, T. C., \& Meltzoff, A. N. (2017). Joint rhythmic movement increases 4-year- old children's prosocial sharing and fairness toward peers. Frontiers in Psychology, 8. doi.org/10.3389/fpsyg.2017.01050.

Rabinowitch, T. C., \& Knafo-Noam, A. (2015). Synchronous rhythmic inter- action enhances children's perceived similarity and closeness towards each other. PLoS ONE, 10, doi: 10.1371/journal.pone.0120878

Ramenzoni, V. C., Sebanz, N., \& Knoblich, G. (2015). Synchronous imitation of continuous action sequences: The role of spatial and topological mapping. Journal of Experimental Psychology: Human Perception and Performance, 41(5), 1209-1222. doi.org/10.1037/xhp0000093

Reddish, P., Fischer, R., \& Bulbulia, J. (2013). Let's dance together: Synchrony, shared intentionality and cooperation. PLOS ONE, 8,1-13. doi:10.1371/journal.pone. 0071182

Richardson, M. J., Marsh, K. L., \& Schmidt, R. C. (2005). Effects of visual and verbal interaction on unintentional interpersonal coordination. Journal of Experimental Psychology: Human Perception and Performance, 31,62-79. doi:10.1037/00961523.31.1.62

Richardson, M. J., Marsh, K. L., Isenhower, R. W., Goodman, J. R. L., \& Schmidt, R. C. (2007). Rocking together: Dynamics of intentional and unintentional interpersonal coordination. Human Movement Science, 26(6), 867-891. doi.org/10.1016/j.humov.2007.07.002

Rizzolatti G (2005) The mirror neuron system and its function in humans. Anat Embryol 210(5):419-421. doi.org/10.1007/s00429-005-0039-Z 
Ropar, D., Greenfield, K., Smith, A. D., Carey, M., \& Newport, R. (2018). Body representation difficulties in children and adolescents with autism may be due to delayed development of visuo-tactile temporal binding. Developmental Cognitive Neuroscience, 29(March 2017), 78-85. doi.org/10.1016/j.den.2017.04.007

Schmidt, R. C., \& Richardson, M. J. (2008). 'Dynamics of interpersonal coordination'. Coordination: Neural, behavioral and social dynamics. 281-308. Springer, Berlin, Heidelberg. doi.org/10.1007/978-3-540-74479-5_14

Schmidt, R. C., Carello, C., \& Turvey, M. T. (1990). Phase transitions and critical fluctuations in the visual coordination of rhythmic movements between people. Journal of Experimental Psychology: Human Perception and Performance, 16,2. doi: 10.1037//0096-1523.16.2.227

Sebanz, N., Bekkering,H., \& Knoblich, G. (2006). Joint action: Bodies and minds moving together. Trends in Cognitive Sciences,10(2), 70-76. doi:10.1016/j.tics.2005.12.009

Stel M, Vonk R., (2010). Empathizing via mimicry depends on whether emotional expressions are seen as real. Eur Psychol, 14:342-350. doi/10.1027/1016-9040.14.4.342

Tomasello, M., Carpenter, M., Call, J., Behne, T., \& Moll, H. (2005). Understanding and sharing intentions: The origins of cultural cognition. Behavioral and Brain Sciences, 28, 675-691. doi: 10.1017/S0140525X05000129.

Trainor, L. J., \& Cirelli, L. (2015). Rhythm and interpersonal synchrony in early social development. Annals of the New York Academy of Sciences, 1337(1), 45-52. doi.org/10.1111/nyas.12649

Tunçgenç, B., \& Cohen, E. (2016a). Interpersonal movement synchrony facilitates pro-social behavior in children's peer-play. Developmental Science, 21(1), 1-9. doi.org/10.1111/desc. 12505

Tunçgenç, B., \& Cohen, E. (2016b). Movement synchrony forges social bonds across group divides. Frontiers in Psychology, 7. doi.org/10.3389/fpsyg.2016.00782

Tunçgenç, B., Cohen, E., \& Fawcett, C. (2015). Rock With Me: The Role of Movement Synchrony in Infants' Social and Nonsocial Choices. Child Development, 86(3), 976984. doi.org/10.1111/cdev.12354

Valdesolo, P., Ouyang, J., \& DeSteno, D. (2010). The rhythm of joint action: synchrony promotes cooperative ability. Journal of Experimental Social Psychology, 46, 693-695. doi.org/10.1016/j.jesp.2010.03.004

van Ulzen, N. R., Lamoth, C. J. C., Daffertshofer, A., Semin, G. R., \& Beek, P. J. (2008). Characteristics of instructed and uninstructed interpersonal coordination while walking side-by-side. Neuroscience Letters, 432(2), 88-93. doi: 10.1016/j.neulet.2007.11.070.

Washburn, A., Kallen, R. W., Coey, C. A., Shockley, K., \& Richardson, M. J. (2015). Harmony from Chaos? Perceptual-Motor Delays Enhance Behavioral Anticipation in Social Interaction. $J$ Exp Psychol Hum Percept Perform, 41(4), 139-148. doi.org/doi:10.1037/xhp0000080 
804 Wiltermuth, S.S., \& Heath, C. (2009). Synchrony and cooperation. Psychological Science, $805 \quad$ 20,1-5. doi:10.1111/ j.1467-9280.2008.02253.x

806 Wing, L., \& Gould, J. (1979). Severe impairments of social interaction and associated abnormalities in children: Epidemiology and classification. Journal of Autism and Developmental Disorders, 9(1), 11-29. doi.org/10.1007/BF01531288 


\section{Appendix A}

Regression table examining how synchrony accuracy predicts social bonding in adults. (outcome variable: Inclusion of Other in Self scale scores).

\begin{tabular}{ccccc}
\hline Predictor & $\boldsymbol{\beta}$ & SE & t value & p value \\
\hline $\begin{array}{c}\text { Synchrony accuracy } \\
\text { (RMS of ITI) }\end{array}$ & .003 & .003 & .96 & .34 \\
\hline
\end{tabular}

809 
Regression table examining how synchrony accuracy predicts social bonding in children (A) with social closeness scale scores as the outcome variable, and (B) with mimicry frequency as the outcome variable.

\begin{tabular}{lcccc}
\hline \multicolumn{1}{c}{ Predictor } & $\boldsymbol{\beta}$ & SE & t value & p value \\
\hline $\begin{array}{l}\text { (A) Synchrony } \\
\text { accuracy (RMS of ITI) }\end{array}$ & .08 & .02 & 4.53 & $<.0001$ \\
\hline $\begin{array}{l}\text { (B) Synchrony accuracy } \\
\text { (RMS of ITI) }\end{array}$ & .001 & .002 & .54 & .59 \\
\hline
\end{tabular}

811 